\title{
Determination of the Effect of Conventional Elastomeric Ligature of Different Ligation techniques versus Nonconventional type on Canine Position using Frictional Mechanics (Typodont Study)
}

Zaid S Tawfek

BDS, MSc (Asst. Lec.)
Dept of Pedod, Orthod, and Prev Dentistry
College of Dentistry, University of Mosul

\begin{abstract}
الخلاصة

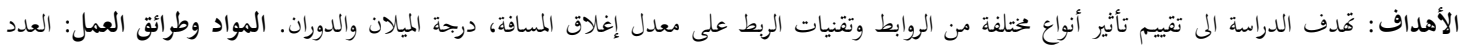

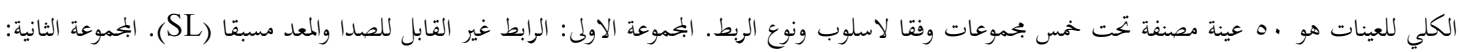

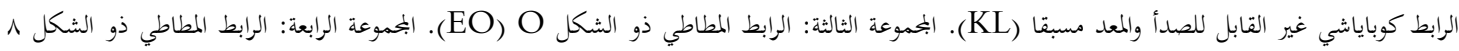

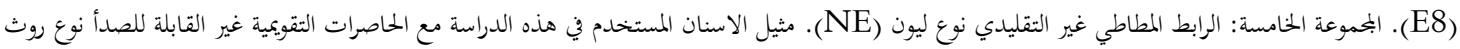

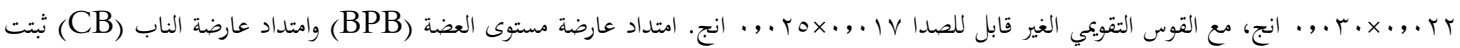

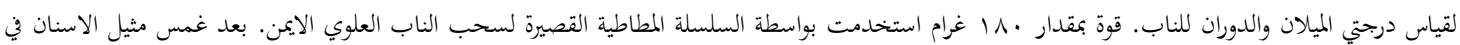

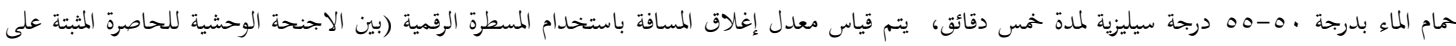

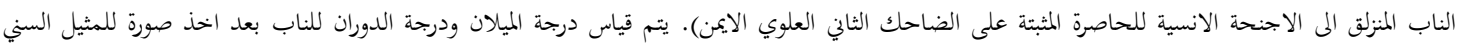

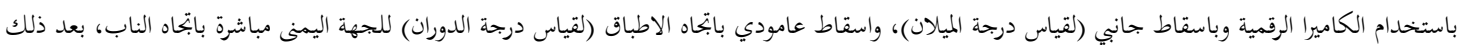

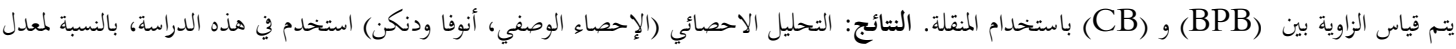

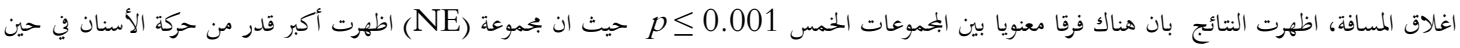

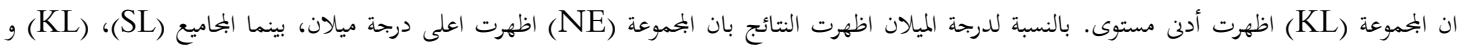

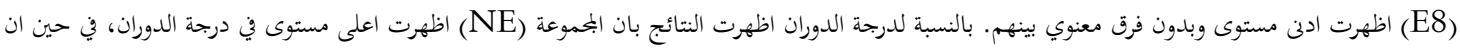

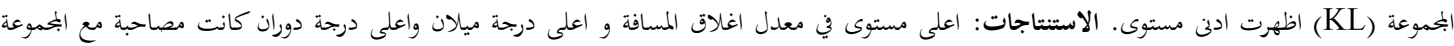

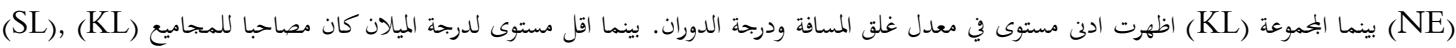

(E8),

\section{ABSTRACT}

Aims: To evaluate the effect of different ligature types and ligation techniques on the rate of space closure, tipping and rotation of canine after sliding. Materials and Methods: The total number of the samples were 50 sample classified under five groups according to the method and type of ligation; the first group: preformed stainless steel ligature wire (SL), the second group: preformed stainless steel kobayashi ligature wire $(\mathrm{KL})$, the third group: elastomeric ligature in figure - $\mathrm{O}(\mathrm{EO})$, the fourth group: elastomeric ligature in figure -8 (E8) and the fifth group: non-conventional Slide Leone elastomeric ligature (NE). A typodont used in this study with preadjusted Roth stainless steel brackets $0.022 \times 0.030$ inch and a stainless steel orthodontic arch wire $0.017 \times 0.025$ inch. A bite plane extension bar (BPB) and canine extension bar (CB) are constructed for the measurement of canine tipping and rotation. A $180 \mathrm{gm}$. of force applied by short continuous elastomeric power chain to retract the right canine. After immersion of the typodont in water bathe with $50-55{ }^{\circ} \mathrm{C}$ for 5 minutes, the rate of space closure were measured in millimeter using vernier (from the distal wings of canine bracket and the mesial wings of the second premolar), the tipping and rotation were measured by taking a photograph to the typodont using digital camera, with transvers projection (for tipping) and occlusal projection (for rotation) from right side (directly toward the right canine), then the angle between (BPB) and (CB) measured directly on the photograph using Protractor. Results: A statistical analysis (descriptive statistics, ANOVA and Duncan Multiple Analysis Range Test) used in this study, for the rate of space closure the results showed that there is a significant difference between the five groups at $p \leq 0.001$, the NE group had the highest amount of tooth movement while KL group had the lowest level, for the tipping; the NE group had the highest level of tipping while SL, KL and E8 groups had the lowest level with a non-significant difference between them, for the rotation; the results showed that NE group had the highest level of rotation, while 
KL group had the lowest level. Conclusions: The higher rate of space closure, the higher rotational degree and the higher tipping degree were associated with NE group, while KL group had shown the lowest rate of space closure and the lowest rotational degree. The lowest degree of tipping was associated with the SL, KL and E8 groups.

Key words: Sliding, nonconventional and conventional elastomeric, typodont, ligation techniques. Tawfek ZS. Determination of the Effect of Conventional Elastomeric Ligature of Different Ligation techniques versus Nonconventional Type on Canine Position Using Frictional Mechanics (Typodont Study). Al-Rafidain Dent J. 2013; 13(2): 358-365.

Received: $27 / 5 / 2012 \quad$ Sent to Referees: $27 / 5 / 2012$

Accepted for Publication: 24/6/2012

\section{INTRODUCTION}

Orthodontic tooth movement during space closure is achieved through two types of mechanics: segmental or sectional mechanics (non frictional system) and sliding mechanics (frictional system) that involve either moving the brackets along an arch wire or sliding arch wire through brackets and tubes. ${ }^{(1)}$ One of main differences between two mechanics is the friction, since sectional mechanics do not involve friction; while friction plays a significant role in sliding space closure. ${ }^{(2)}$ Friction is the resistance to motion when an object moves tangentially against another. ${ }^{(3,4)}$ During orthodontic space closure with sliding mechanics, frictional force is generated at the bracket arch wire interface and has a tendency to inhibit the desired tooth movement. ${ }^{(5)}$ During orthodontic treatment with fixed appliances, frictional forces should be kept to a minimum so that lower levels of force can be applied to obtain an optimal biological response for effective tooth movement. ${ }^{(6,7)}$ Many studies have evaluated the factors that influence frictional resistance: bracket and wire materials, surface conditions of arch wires and bracket slot, wire section, torque at the wire-bracket interface, type and force of ligation, use of self-ligating brackets, interbracket distance, saliva, and influence of oral functions. ${ }^{(8-11)}$ The method of arch wire ligation would appear to be an important determinant in the generation of friction, ${ }^{(12)}$ Ligature elastic either placed around the bracket in a figure-O pattern or in a figure8 pattern, this ligation method will increase the frictional force about $80 \% .^{(13)} \mathrm{New}$ low-friction ligatures have been introduced, similar to elastic ligatures, but with an anterior part that is more rigid and similar to the mechanical device of self-ligating brackets. ${ }^{(14)}$

The "nonconventional" elastomeric ligature (low-friction ligatures) is used on conventional brackets to produce low levels of frictional resistance in treatment mechanics with the preadjusted appliance. Once the unconventional ligature is applied on the bracket, the interaction between the ligature and the slot forms a "tube-like" structure, which allows the arch wire to slide freely and to produce its effects more readily on the dentoalveolar component. ${ }^{(15)}$

The aims of the study were to evaluate the effect of different ligature types and ligation techniques on the rate of space closure, tipping and rotation of canine after sliding.

\section{MATERIALS AND METHODS}

The typodont used in this study prepared according to manufacturer's instructions (Ormco), the preadjusted Roth stainless steel brackets $0.022 \times 0.030$ inch (Dentaurum, Germany) were fixed on the metallic typodont teeth by using epoxy steel adhesive, ${ }^{(16)}$ the vertical accuracy of brackets were checked by using the bracket positioning gauge, ${ }^{(17)}$ the preadjusted Roth stainless steel molar bands were also fixed to the metallic typodont first molar tooth by using Zinc phosphate orthodontic cement. A class II typodont wax form was used, so alignment of the teeth was done by using arch wires started from 0.014 inch $\mathrm{NiTi}$ and gradually upgraded till we reach $0.017 \times 0.025$ inch Stainless steel arch wire, ${ }^{(18,19)}$ The first right premolar was removed from the typodont to allow a space for canine sliding. Acrylic bite plane was constructed by taking a primary alginate impression for the aligned teeth then pouring by plaster materials after that a special tray was made and a final impression taken with alginate to construct a master cast, then wax materials was applied in a way that cover the occlusal, incisal, palatal, labial and lingual surface of the teeth (except the canine and the first premolar extraction 
space), then the wax replaced by hot cure acrylic resin. ${ }^{(20)}$

A special two bars were constructed, a bite plane extension bar (BPB) and canine extension bar (CB) (Figure 1), the BPB is a L-shape bar made from $0.016 \times 0.016$ inch stainless steel rectangular wire, the short arm is inserted in the simulated rugae area in the palatal midline of the acrylic bite plane, this bar emerges upward for 10 $\mathrm{mm}$ then it bends and extends facially 20 $\mathrm{mm}$ to make right angle with $\mathrm{CB},{ }^{(20)}$ the $\mathrm{CB}$ were also constructed from $0.016 \times 0.016$ inch stainless steel rectangular wire in a form of L-shape, the short arm is welded to the distal aspect of the right canine (the tooth to be slides), this arm extended upward incisally $10 \mathrm{~mm}$ then it bends at right angle and extended anteriorly $20 \mathrm{~mm}$ and $5 \mathrm{~mm}$ over the tip of canine cusp and under the BPB by about 5 $\mathrm{mm}$. such two bars are used as a guide to determine the position of canine after sliding regarding the degree of tipping and rotation, this method is a modification of Huffman and Way procedure. ${ }^{(21)}$ A special wooden table was constructed with two metallic bases, one to fix the digital camera (vertically for rotational measurements and horizontally for tipping measurements), and the other to fix the typodont in a way to allow a standard method for taking a photograph before and after each sliding.

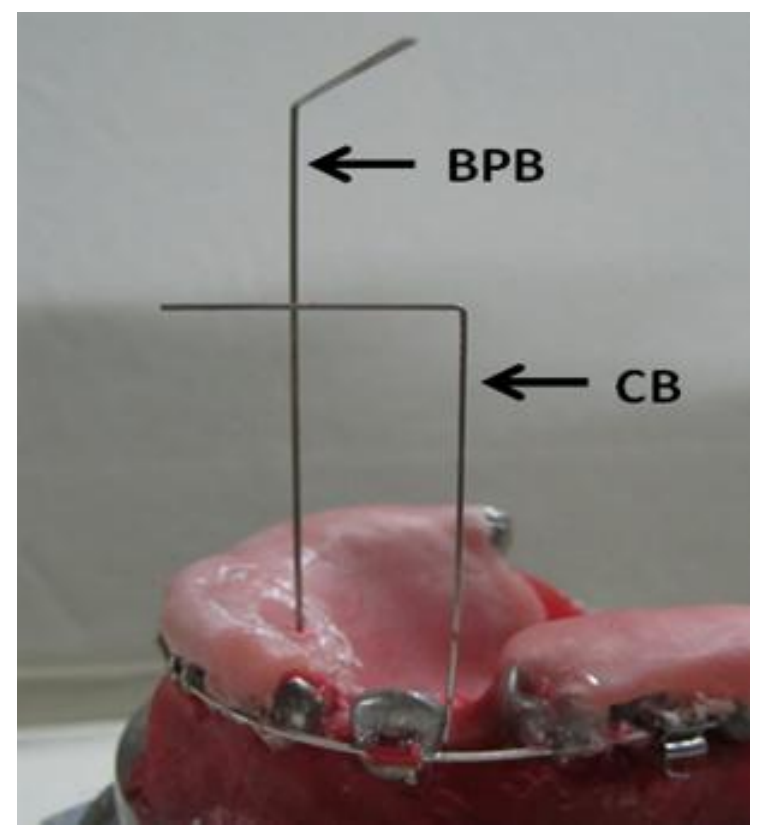

Figure (1): Canine extension bar (CB) and bite plane extension bar (BPB).

The samples of this study divided into five groups (10 samples for each group), with a different ligation types, methods and techniques. The first group: Preformed stainless steel ligature wire (SL) (gauge 0.010 inch), Dntaurum, Germany. The second group: Preformed stainless steel kobayashi ligature wire (KL) (gauge 0.012 inch), Dntaurum, Germany. The third group: Elastomeric ligature in figure $-\mathrm{O}$ (EO), Dntaurum, Germany. The fourth group: Elastomeric ligature in figure -8 (E8) (Figure 2), Dntaurum, Germany. The fifth group: Non-conventional Slide Leone elastomeric ligature (NE) (Figure 3), Lancer, USA. for the (SL) and (KL), the wire twisted 8 turns by using Mathieu needle holder (Dentaurum, Germany) and then cut the access leaving small part of it tucked behind the arch wire, ${ }^{(22)}$ this procedure was repeated after each reading.

The retraction of canine was done by using a short continuous elastomeric power chain attached distally to the hook of the first molar band and mesially to the canine hook, the force that applied is about $180 \mathrm{gm}$ according to manufactures instruction (Ormco), measured by using a tension 
gauge. The typodont was immersed in a water bath (HAAKE - England) with 50$55{ }^{0} \mathrm{C}$ for 5 minutes then removed and immersed directly in a tap water $\left(5^{0}-\right.$ $\left.10^{\circ} \mathrm{C}\right){ }^{(23)}$

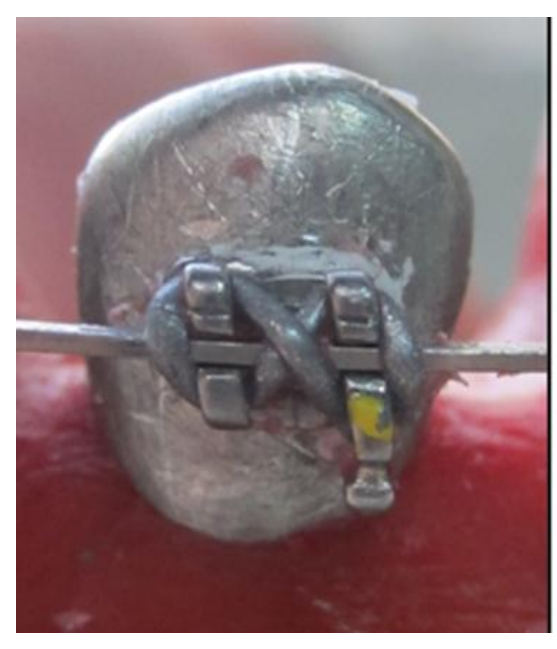

Figure (2): Elastomeric ligature in figure 8 .
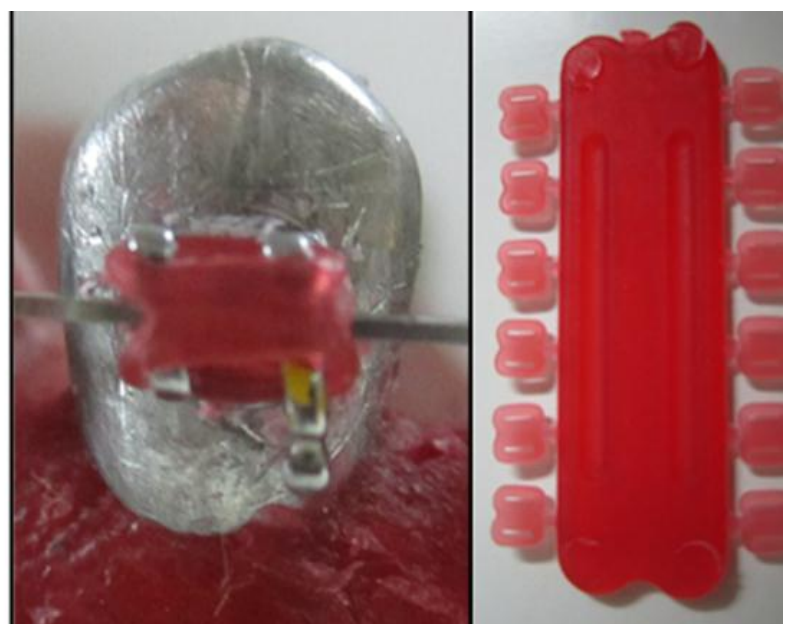

Figure (3): Non-conventional slide leon elastomeric ligature.
Canine position measurements before sliding:

Before starting sliding of right canine into first premolar site (for the all readings), the following was checked; first: all the teeth situated in well aligned position. Second: the distance between the distal wings of canine bracket and the mesial wings of the second premolar were measured in millimeter by using digital vernier before each sliding (available space). Third: the angle between BPB and CB should be $90^{\circ}$ from both horizontal and vertical direction; this angle is considered as $\mathrm{CB}$ original angle.

Canine position measurements after sliding:

The distance between the distal wings of canine bracket and the mesial wings of the second premolar were again measured by using digital vernier after each sliding (remaining space), then the rate of space closure was measured in millimeter (by subtraction of the remaining space from the available space).

The canine's tipping degree after movement was measured by taking a photograph to the typodont using digital cam- era, with transvers projection from right side (directly toward the right canine) where the angle between BPB and CB is exposed and then can be measured directly on the photograph using Protractor, this angle is considered as CB inclination angle, then the canine's tipping was measured by subtraction of the $\mathrm{CB}$ inclination angle from the $\mathrm{CB}$ original angle.

The canine's rotation degree after movement was measured by taking a photograph to the typodont using digital camera, with vertical projection from occlusal side (directly toward the right canine) where the angle between $\mathrm{BPB}$ and $\mathrm{CB}$ is exposed and then can be measured directly on the photograph using Protractor, this angle is considered as CB rotational angle, then the canine's rotation was measured by subtraction of the $\mathrm{CB}$ rotational angle from the $\mathrm{CB}$ original angle.

\section{RESULTS}

The descriptive statistics (mean, standard deviations, standard error, minimum and maximum values) for the rate of space closure, tipping and rotation of the five groups of ligation are listed in the Table (1). 
Table (1 ): Descriptive analysis (mean, standard deviations, standard error, minimum and maximum values) for the rate of space closure, tipping and rotation of the five groups of ligation.

\begin{tabular}{cccccccc}
\hline & & No. & Mean & SD & SE & Min. & Max. \\
\hline \multirow{5}{*}{ Rate of } & SL & 10 & 3.5000 & .52705 & .16667 & 3.00 & 4.00 \\
Space & EL & 10 & 1.4000 & .51640 & .16330 & 1.00 & 2.00 \\
closure & E8 & 10 & 5.3000 & .82327 & .26034 & 4.00 & 6.00 \\
& NE & 10 & 2.1000 & .73786 & .23333 & 1.00 & 3.00 \\
& Total & 50 & 7.4000 & .69921 & .22111 & 6.00 & 8.00 \\
& SL & 10 & 4.9400 & 2.2982 & .32502 & 1.00 & 8.00 \\
\hline \multirow{5}{*}{ Tipping } & KL & 10 & 4.9000 & .63246 & .20000 & 4.00 & 6.00 \\
& EO & 10 & 6.4000 & .73786 & .23333 & 4.00 & 6.00 \\
& E8 & 10 & 5.5000 & .70711 & .16330 & 6.00 & 7.00 \\
& NE & 10 & 13.000 & 1.0540 & .33333 & 5.00 & 7.00 \\
& Total & 50 & 6.9200 & 3.2061 & .45341 & 4.00 & 15.00 \\
& SL & 10 & 6.2000 & .63246 & .20000 & 5.00 & 7.00 \\
& KL & 10 & 3.0000 & .66667 & .21082 & 2.00 & 4.00 \\
& EO & 10 & 21.6000 & .69921 & .22111 & 21.00 & 23.00 \\
& E8 & 10 & 9.7000 & .67495 & .21344 & 9.00 & 11.00 \\
& NE & 10 & 35.1000 & 1.28668 & .40689 & 33.00 & 37.00 \\
& Total & 50 & 15.1200 & 11.9533 & 1.6904 & 2.00 & 37.00 \\
\hline
\end{tabular}

No: number; SD: standard deviation; SE: standard error; Min: minimum value; Max: maximum. SL: Preformed stainless steel ligature wire; KL: Preformed stainless steel kobayashi ligature wire; EO: Elastomeric ligature in figure - o; E8: Elastomeric ligature in figure - 8; NE: Non-conventional slide leone elastomeric ligature.

Rate of space closure:

The ANOVA test for the rate of space closure showed that there is a significant difference between the five groups at $p \leq$ 0.001 as shown in the Table (2). The Duncan Multiple Analysis Range Test showed that NE group had the highest amount of tooth movement while KL group had the lowest level, while the remaining groups distributed on statistical levels between the highest and lowest groups with a significant differences at $p \leq 0.001$ as shown in the Table (3).

Table (2): ANOVA test for the rate of space closure.

\begin{tabular}{cccccc}
\hline & Sum of Squares & df & Mean Square & F & Sig.* \\
\hline Between Groups & 238.520 & 4 & 59.630 & 132.185 & .000 \\
Within Groups & 20.300 & 45 & .451 & & \\
Total & 258.820 & 49 & & & \\
\hline
\end{tabular}

df: degree of freedom. $*$ Significant difference at $p \leq 0.0001$.

Table (3): Duncan's multiple range test for the rate of space closure.

\begin{tabular}{cccc}
\hline Groups & No. & Mean* \pm SE & Duncan Groups** \\
\hline KL & 10 & $1.4000 \pm .16330$ & $\mathrm{~A}$ \\
E8 & 10 & $2.1000 \pm .23333$ & $\mathrm{~B}$ \\
SL & 10 & $3.5000 \pm .16667$ & $\mathrm{C}$ \\
EO & 10 & $5.3000 \pm .26034$ & $\mathrm{D}$ \\
NE & 10 & $7.4000 \pm .22111$ & $\mathrm{E}$ \\
\hline
\end{tabular}

SL: Preformed Stainless Steel ligature wire; KL: Preformed Stainless Steel Kobayashi ligature wire; EO: Elastomeric ligature in figure - o; E8: Elastomeric ligature in figure - 8; NE: Nonconventional Slide Leone elastomeric ligature. No: number ;*Mean measurement unit in millimeter **Different litters mean significant difference at $p \leq 0.0001$. 
Tipping:

The ANOVA test for the tipping showed that there is a significant difference between the five groups at $p \leq 0.001$ as shown in the Table (4). The Duncan Multiple Analysis Range Test showed that
NE group had the highest level of tipping while SL, KL and E8 groups had the lowest level with a non-significant difference between them at $p \leq 0.001$ as shown in the Table (5).

Table (4): ANOVA test for the Tipping.

\begin{tabular}{cccccc}
\hline & Sum of Squares & df & Mean Square & F & Sig.* \\
\hline Between Groups & 478.280 & 4 & 119.570 & 211.837 & .000 \\
Within Groups & 25.400 & 45 & .564 & & \\
Total & 503.680 & 49 & & & \\
\hline
\end{tabular}

df: degree of freedom. *Significant difference at $p \leq 0.0001$.

Table (5): Duncan's multiple range test for the Tipping.

\begin{tabular}{cccc}
\hline Groups & No. & Mean* \pm SE & Duncan Groups** \\
\hline SL & 10 & $4.8000 \pm .20000$ & $\mathrm{~A}$ \\
KL & 10 & $4.9000 \pm .23333$ & $\mathrm{~A}$ \\
E8 & 10 & $5.5000 \pm .22361$ & $\mathrm{~A}$ \\
EO & 10 & $6.4000 \pm .16330$ & $\mathrm{~B}$ \\
NE & 10 & $13.0000 \pm .33333$ & $\mathrm{C}$ \\
\hline
\end{tabular}

SL: Preformed Stainless Steel ligature wire; KL: Preformed Stainless Steel Kobayashi ligature wire; EO: Elastomeric ligature in figure - o; E8: Elastomeric ligature in figure - 8; NE: Non-conventional Slide Leone elastomeric ligature. No: number ;*Mean measurement unit in degree. ${ }^{* *}$ Different litters mean significant difference at $p \leq 0.0001$.

\section{Rotation:}

The ANOVA test for the rotation showed that there is a significant difference between the five groups at $p \leq 0.001$ as shown in the Table (6). The Duncan Multiple Analysis Range Test showed that NE group had the highest level of rotation, while KL group had the lowest level, while the remaining groups are distributed between the highest and lowest groups with a significant differences at $p \leq 0.001$ as shown in the Table (7).

Table (6): ANOVA test for the Rotation.

\begin{tabular}{cccccc}
\hline & Sum of Squares & df & Mean Square & F & Sig.* \\
\hline Between Groups & 6970.280 & 4 & 1742.570 & 2529.537 & .000 \\
Within Groups & 31.000 & 45 & .689 & & \\
Total & 7001.280 & 49 & & & \\
\hline
\end{tabular}

df: degree of freedom. *Significant difference at $p \leq 0.0001$.

Table (7): Duncan's multiple range test for the Rotation.

\begin{tabular}{cccc}
\hline Groups & No. & Mean* \pm SE & Duncan Groups** \\
\hline KL & 10 & $3.0000 \pm .21082$ & $\mathrm{~A}$ \\
SL & 10 & $6.2000 \pm .20000$ & $\mathrm{~B}$ \\
E8 & 10 & $9.7000 \pm .21344$ & $\mathrm{C}$ \\
EO & 10 & $21.6000 \pm .22111$ & $\mathrm{D}$ \\
NE & 10 & $35.1000 \pm .40689$ & $\mathrm{E}$ \\
\hline
\end{tabular}

SL: Preformed Stainless Steel ligature wire; KL: Preformed Stainless Steel Kobayashi ligature wire; EO: Elastomeric ligature in figure - o; E8: Elastomeric ligature in figure - 8; NE: Nonconventional Slide Leone elastomeric ligature. No: number ;*Mean measurement unit in degree. ${ }^{*}$ Different litters mean significant difference at $p \leq 0.0001$. 


\section{DISCUSSION}

Regarding the amount of tooth movement after canine sliding, the result of this study showed that the NE group had the highest amount of tooth movement and this is due to low frictional resistance associated with the using of non-conventional elastomeric ligature, this is agree with Baccetti and Franchi ${ }^{(23)}$ who stated that when the non-conventional elastomeric ligature is applied on the bracket, the interaction between the ligature and the slot form a tube-like structure which allows the arch wire to slide freely and achieve a large amount of tooth movement. While the lowest amount of tooth movement that occurs with KL group may be attributed to the highly frictional force that is generated between the bracket and arch wire due to a large diameter and stiff kobayashi ligature wire (gauge 0.012 inch). Regarding the degree rotation after canine sliding the results showed that NE group had the highest degree of rotation and this is may be due to the design of non-conventional elastomeric ligature that makes it fit on bracket slot without any pressure on arch wire (tube like structure) that allowing the bracket to rotate ${ }^{(23,15)}$ while KL group shown a lowest degree of rotation after canine sliding and this is because that the large diameter (gauge 0.012 inch) kobayashi ligature wire that press the arch wire tightly against the bracket provide enough force to produce the adequate anti-rotation moment. Regarding the degree tipping after canine sliding, this study showed that all the groups had a different degrees of tipping, and this is agree with Ehsania et al., ${ }^{(24)}$ who mentioned that the tipping is a constant phenomenon during sliding and it always occurs when orthodontic force is applied to the tooth. The highest degree of tipping that associated with NE group may be attributed to high rotational degree that makes part of the arch wire completely out of the slot at the distoocclusal and distogingival wings, while the SL, KL and E8 groups had the lowest degree of tipping with a non-significant difference between them, and this is may be due to proper orientation and position of canine during sliding resulting from the pressure of ligation, and also the same bracket width, arch wire cross section and the same point of force application.

\section{CONCLUSIONS}

It was concluded that the higher rate of space closure, the higher rotational degree and the higher tipping degree were associated with non-conventional elastomeric ligature (NE group), while kobayashi ligature $(\mathrm{KL})$ group was shown the lowest rate of space closure and the lowest rotational degree. While the lowest degree of tipping was associated with the SL, KL and E8 groups.

\section{REFERENCES}

1. Gjessing P. Biomechanical design and clinical evaluation of a new canineretraction spring. Am J Orthod Dentofaclial Orthop. 1985; 87(5): 353-362.

2. Tecco S, Festa F, Caputi S, Traini T, Di Iorio D, D'Attilio M. Friction of conventional and self-ligating brackets using a 10 bracket model. Angle Orthod. 2005; 75(6):1041-5.

3. Cicciafesta V, Sfondini MF, Ricciardi A, Scribante A, Klersy C, Auricchio F. Evaluation of friction of stainless steel and esthetic self-ligating brackets in various bracket-archwire combinations. Am J Orthod Dentofacial Orthop. 2003; 124(4): 395-402.

4. Cash A, Curtis R, Garrigia-Majo D, McDonald F. A comparative study of the static and kinetic frictional resistance of titanium molybdenum alloy archwires in stainless steel brackets. Eur J Orthod. 2004; 26 (1): 105-111.

5. Angolkar PD, Kapila S, Duncanson MG Jr, Nanda RS. Evaluation of friction between ceramic brackets and orthodontic wires of four alloys. Am J Orthod Dentofacial Orthop. 1990; 98: 499-506.

6. Ogata RH, Nanda RS, Duncanson MG Jr, Sinha PK, Currier GF. Frictional resistances in stainless steel bracket-wire combinations with effects of vertical deflections. Am J Orthod Dentofacial Orthop. 1996; 109(5): 535-542.

7. Andreasen GF, Quevedo FR. Evaluation of frictional forces in the $0.022 \times 0.028$ edgewise bracket in vitro. $J$ Biomech. 1970; 3(2): 151-160

8. Kapila S, Angolkar PV, Duncanson MG, Nanda RS. Evaluation of friction between edgewise stainless steel brackets and orthodontic wires of four alloys. Am J Orthod 
Dentofacial Orthop. 1990; 98 (2): $117-$ 126.

9. Rose CM, Zernik JH. Reduced resistance to sliding in ceramic brackets. J Clin Orthod. 1996; 30(2): 78-84.

10.Braun S, Bluestein M, Moore BK, Benson G. Friction in perspective. Am J Orthod Dentofacial Orthop. 1999; 115(6): 619627.

11.Hain M, Dhopatkar A, Peter R. The effect of ligation method on friction in sliding mechanics. Am J Orthod Dentofacial Orthop. 2003; 123(4): 416-22.

12.Sims AP, Waters NE, Birnie DJ, Pethybrige RJ. A comparison of the forces required to produce tooth movement in vitro using two self-ligating and a preadjusted bracket employing two types of ligation. Eur J Orthod. 1993; 15(5): 377-85.

13. Tecco S, Di Iorio D, Cordasco G, Verrocchi I, Festa F. An in vitro investigation of the influence of self-ligating brackets, low friction ligatures, and arch wire on frictional resistance. Eur J Orthod. 2007; 29(4): 390-397.

14.Baccetti T, Franchi L, Camporesi M. Forces in the presence of ceramic versus stainless steel brackets with unconventional vs conventional ligatures. Angle Orthod. 2008; 78(1): 120-124.

15.Lima V, Coimbra M, Derech C. Frictional forces in stainless steel and plastic brackets using four types of wire ligation. Dental Press J Orthod. 2010; 15(2): 82-86.

16. MuLaughlin RP, Bannett JC. Bracket placement with the preadjusted appliance. $J$ Clin Orthod. 1995; 29(5): 302-311.
17.Elsheikh MM, Godfreg K, Manosudprasit M, Viwattanatipa N. A forsus distalizer: A pilot typodont study. Am J Orthod Dentofacial Orthop. 2004; 7(2): 107-116.

18.Eliades T, Boourauel C. Intraoral aging of orthodontic materials: the picture we miss and its clinical relevance. Am J Orthod Dentofacial Orthop. 2005; 127(4): 403412.

19.Al-Shahery WGh. Upper canine retraction using stainless steel and nickel titanium coil springs (in vitro study). Msc. Thesis. Collage of Dentistry, University of Mosul. 2010.

20.Huffman DJ, way DC. A clinical evaluation of tooth movement along arch wires of two different sizes. Am J Orthod Dentofacial Orthop. 1983; 83(6): 453-459.

21. Hain M, Dhopatkar A, Rock P. The effect of ligation method on friction in sliding mechanics. Am J Orthod Dentofacial Orthop. 2003; 123(4):416- 22.

22. Ogura M, Yamagata K, Kubota S, Kim JH, Kuroe K, Ito G. Comparison of tooth movements using Friction-Free and preadjusted edgewise bracket systems. J Clin Orthod. 1996; 30(6): 325-330.

23. Baccetti T, Franchi L. Friction produced by types of elastomeric ligatures in treatment mechanics with the preadjusted appliance. Angle Orthod. 2006; 76(1): 211-6.

24.Ehsania S, Mandichb MA, El-Bialyc TH, Mirc CF. Frictional resistance in selfligating orthodontic brackets and conventionally ligated brackets. Angle Orthod. 2009; 79(3): 592-601. 